\title{
Application of Recent International Epidemiological Guidelines to a Prospective Study of the Incidence of First Seizures, Newly-Diagnosed Epilepsy and Seizure Mimics in a Defined Geographic Region in Ireland
}

\author{
Eimer M. Maloney ${ }^{a-c}$ Elijah Chailad Éilis J. O'Reilly ${ }^{b, e}$ Daniel J. Costello ${ }^{a, c}$ \\ ${ }^{a}$ Department of Neurology, Cork University Hospital, Cork, Ireland; ${ }^{b}$ School of Public Health, University College \\ Cork, Cork, Ireland; ' ${ }^{c}$ College of Medicine and Health, University College Cork, Cork, Ireland; d Department of \\ Neurology, University Hospital Limerick, Limerick, Ireland; e Department of Nutrition, Harvard TH Chan School of \\ Public Health, Boston, MA, USA
}

\section{Keywords}

Epilepsy · Incidence · Epidemiological protocol

\begin{abstract}
Studies adherent to international guidelines and epilepsy classification are needed to accurately record the incidence of isolated seizures, epilepsy and seizure-mimics within a population. Because the diagnosis of epilepsy is largely made through clinical assessment by experienced physicians, seizures and epilepsy are susceptible to misdiagnosis. Previous epidemiological studies in epilepsy have not captured "seizure mimics". We therefore sought to quantify the incidence of isolated seizures, epilepsy and seizure-mimics using the International League Against Epilepsy (ILAE) classification system. In this study multiple overlapping methods of case ascertainment were applied to a defined geographic region from January 1 to March 31, 2017 to identify all patients presenting with first seizures (provoked and unprovoked), new diagnoses of epilepsy and seizure mimics. Over a 3 month period, from a population of 542,869 adults and children, 442 potential presentations were identified,
\end{abstract}

and 283 met the inclusion criteria. Radiology databases were the source of the largest number of individual cases $(n=153$, $54 \%$ ), while electroencephalogram (EEG) databases were the source of the highest number of unique-to-source cases (those not identified elsewhere, $n=60,21 \%$ ). No single case was picked up in every method of ascertainment. Among the 283 included presentations, 38 (13\%) were classed as first provoked seizures, 27 (10\%) as first unprovoked seizures, 95 (34\%) as new diagnosis of epilepsy and 113 (40\%) as seizure mimics. Ten (3\%) presentations were indeterminate. We present and apply a rigorous study protocol for investigation of the incidence of first seizures, new diagnosis of epilepsy and seizure mimics in a geographically defined region which is adherent to recently published international guidelines for epidemiologic studies and epilepsy classification. We highlight the challenges in making a diagnosis of new-onset epilepsy in patients presenting with a first seizure using the current ILAE definition of epilepsy, when epilepsy can be diagnosed in situations where the treating physician anticipates the risk of further seizures exceeds $60 \%$.

(c) 2019 S. Karger AG, Base

\section{KARGER}

(C) 2019 S. Karger AG, Basel

E-Mail karger@karger.com

www.karger.com/ned
Eimer M. Maloney 


\section{Introduction}

Epilepsy is a disorder of the brain characterized by an enduring predisposition to generate epileptic seizures and by the neurobiologic, cognitive, psychologi$\mathrm{cal}$, and social consequences of this condition [1]. Operationally, epilepsy can be diagnosed in 3 circumstances: (i) at least 2 unprovoked (or reflex) seizures occurring $>24$ h apart, (ii) one unprovoked (or reflex) seizure and a probability of further seizures similar to the general recurrence risk (at least 60\%) after 2 unprovoked seizures, occurring over the next 10 years, and (iii) diagnosis of an epilepsy syndrome [2]. The second situation is relatively common but difficult to apply in real life as judging that an individual patient has a $60 \%$ risk of further seizures is challenging. Epilepsy poses a substantial economic burden for health care systems, individuals and their families through direct costs of treatment and indirect costs such as loss of productivity and employment [3]. Epidemiological studies are necessary to define the full public health burden of epilepsy within a population, to provide information needed for early detection and treatment, and to set public health and health care priorities [4]. Assessment of patients presenting with first seizures is critical to differentiating epileptic seizures from other conditions that resemble epileptic seizures ("seizure mimics"). Although first seizures and epilepsy are susceptible to misdiagnosis, to our knowledge there are no published epidemiological studies on seizure mimics.

There are significant disparities in reported prevalence and incidence of epilepsy worldwide. While some variation may be related to factors such as socioeconomic class, access to healthcare, stigmatization and exposure to environmental risk factors [5], a significant contributor to the variation in reported incidence and prevalence may be due to heterogeneous methodologies used across studies. A recent meta-analysis of 48 incidence studies noted significant heterogeneity between studies and estimated the pooled annual cumulative incidence of epilepsy as 67.77 per 100,000 persons (95\% CI 56.6-81.0) with one outlier report of 189.96 per 100,000 [6]. Methods of case ascertainment in published studies range from door-todoor population surveys, administrative database searches and surveys of neurology referral centers [7-9], thus providing variations in inclusion criteria and accuracy of case ascertainment. Many studies employ retrospective case ascertainment and lack of detailed diagnostic information often prohibits accurate classification of seizure and epilepsy type.
In 2011, in an effort to promote consistency in definitions and methods used in epidemiological studies and to facilitate comparisons between populations, the International League Against Epilepsy (ILAE) proposed standards for epidemiologic studies and surveillance of epilepsy [4]. These guidelines emphasize the importance of using multiple overlapping methods of case ascertainment to maximize the sensitivity of case ascertainment. Furthermore, Standards of Reporting of Neurological Disorders guidelines for reporting of incidence and prevalence studies in neuroepidemiology have been developed to facilitate better reporting of published data and allow comparisons between studies [10]. When applied to future studies, these guidelines will enhance populationbased epidemiological studies and encourage the collection of data useful for the promotion of public health. Finally, the ILAE has recently commissioned updated classifications systems for both seizures and epilepsy types $[11,12]$. These classification systems are userfriendly and allow classification of seizure and epilepsy type by taking into account results of electroencephalogram (EEG) and imaging investigations. To our knowledge, this is the first epidemiological study to incorporate these classifications.

In Ireland, it has been estimated that 10 per 1,000 persons aged 18 years and older have a self-reported lifetime prevalence of epilepsy [13]. This study is the first to investigate the incidence of new onset seizures, epilepsy and seizure mimics in Ireland and, to our knowledge, the first to present epidemiological data on seizure mimics. We present our study protocol, adherent to ILAE and Standards of Reporting of Neurological Disorder guidelines, and findings for the first 3 months of data collection.

\section{Materials and Methods}

This study was carried out in the geographically defined area of Cork city and county over the course of the calendar year 2017, with an estimated total population of 542,868 persons based on a census in 2016. Herein we present our study protocol and, to demonstrate its application, the results of case ascertainment for the first 3 months of data collection, January 1, 2017 to March 31, 2017.

All acute medical hospitals were included with the exception of one solely private hospital that does not employ an on-site consultant neurologist. Acute seizures presenting to the medical assessment unit of that hospital are transferred to the tertiary university hospital in the city. The remaining 7 acute hospitals included in this study were as follows: 2 tertiary referral city center university hospitals, 2 regional secondary level hospitals, 1 solely private city center hospital which accepts acute referrals and employs 2 consultant neurologists, 2 city center hospitals with facilities for rehabilitation of geriatric patients who have recently been acutely ad- 
Table 1. Examples of provoked seizures in association with disruption of the structural or functional integrity of the brain, adapted from the International League Against Epilepsy Standards for Epidemiologic Studies [4]

\begin{tabular}{|c|c|c|}
\hline Cause $[14]$ & Period of occurence & Notes/exceptions \\
\hline Cerebrovascular disease [15-17] & First 7 days & \\
\hline Traumatic brain injury $[17,18]$ & First 7 days & $\begin{array}{l}\text { Included intracranial surgery. } \\
\text { Longer intervals are acceptable for subdural hematoma in } \\
\text { the absence of known trauma or at first identification of } \\
\text { hematoma. Subsequent seizures are unprovoked }\end{array}$ \\
\hline CNS infection $[14,17]$ & First 7 days & $\begin{array}{l}\text { Included seizures occuring after } 7 \text { days in patients with } \\
\text { persistent clinical and/or laboratory signs of infection }\end{array}$ \\
\hline Cerebral tuberculoma [14] & During treatment & Seizures occuring after successful treatment are unprovoked \\
\hline Brain abscess $[14]$ & During treatment & Seizures occuring after successful treatment are unprovoked \\
\hline HIV infection [14] & $\begin{array}{l}\text { Acute infection or severe } \\
\text { metabolic disturbance }\end{array}$ & $\begin{array}{l}\text { Seizures occuring in the absence of opportunistic CNS } \\
\text { infection or severe metabolic disturbance are unprovoked }\end{array}$ \\
\hline Arterovenous malformation [14] & $\begin{array}{l}\text { In the presence of acute } \\
\text { hemorrhage }\end{array}$ & All other seizures are unprovoked \\
\hline
\end{tabular}

Multiple sclerosis [14]

First presenting symptom

within 7 days of relapse

Autoimmune disease [14]

Signs or symptoms of activation

mitted elsewhere for example, ortho-geriatrics rehabilitation. This study also included community sources of case ascertainment as follows: all general practitioners (GPs) in Cork city and county who are registered with the Irish Medical Directory, all nursing homes and residential care centers registered with the Health Information and Quality Authority, and Epilepsy Ireland, the community based patient information and advocacy group.

\section{Inclusion and Exclusion Criteria}

We included all patients who had a suspected first seizure or new diagnosis of epilepsy from January 1 to December 31, 2017 whose registered address as per the hospital-based demographic information was within Cork city or county (defined area). The working diagnosis of "seizure" had to be explicitly documented in medical correspondence during the patient's assessment typically by a GP in the community, an emergency department triage nurse or physician, or senior hospital-based physician. Any patient, normally resident in the defined area, who had a suspected seizure during 2017 but presented to a hospital outside the area was included. These patients were identified through survey of GPs and neurologists in Cork city and county, where their ongoing care and follow up was based. All patients with an address outside of the defined area were excluded.

We gathered information on all patients whose first presentation to medical services with a query of seizure occurred during 2017. In some cases, the date of the first clinical event was prior to the 2017 calendar year, but medical attention was first sought in 2017 , for example, when a patient has a history of recurrent stereotyped events, but the possibility of these events being seizures had not yet been explicitly stated, or medical attention was not sought. Similarly, we continued to monitor a number of case ascertain-

Methodology of a Prospective Incidence Study on Epilepsy, Seizures and Mimics ment sources (rapid access seizure clinic [RASC] referrals, EEG and radiology databases) up to March 31, 2018 in order to complete the classification of patients who first presented in late 2017. By restricting our count to first presentation during 2017, we will avoid over- or under-ascertainment in the 2017 calendar year, and the number of first presentations classified will estimate the true incidence of each clinical subcategory.

As per the ILAE epidemiologic guidelines [4], febrile seizures in children ( 3 months to 6 years old) and neonatal seizures $(<28$ days old) were excluded from this study as they are felt to be separate from epilepsy per se. With regard to acute provoked seizures, we noted the incidence of their occurrence as a specific research question, but they were separated from epilepsy in the final analysis.

\section{ILAE Definitions}

i) An epileptic seizure is a transient occurrence of signs and/or symptoms due to abnormal excessive or synchronous neuronal activity in the brain [1].

ii) Epilepsy is a disease of the brain defined by any of the following conditions: (i) at least 2 unprovoked (or reflex) seizures occurring $>24 \mathrm{~h}$ apart (ii) one unprovoked (or reflex) seizure and a probability of further seizures similar to the general recurrence risk (at least 60\%) after 2 unprovoked seizures, occurring over the next 10 years, or (iii) diagnosis of an epilepsy syndrome [2].

iii) Acute provoked seizures are epileptic seizures which occur in close temporal association with an acute systemic, metabolic or toxic insult or in association with an acute central nervous system insult [4]. The interval between the insult and the seizure may vary according to the underlying clinical condition (Table 1). With regard to alcohol withdrawal seizures, the seizure must have oc- 
curred within 7-48 h of the last drink to be classified as an alcohol withdrawal seizure [14]. Alternatively, any patient with a history of alcohol abuse and acute provoked seizures from alcohol, who developed their first seizure independent from alcohol within the study period (and was therefore commenced on anti-epileptic drug [AED]) was counted as a "new diagnosis of epilepsy". Seizures in the setting of acute alcohol intoxication due to extremely high quantities consumed and seizures in association with withdrawal of benzodiazepines were classified as acute provoked seizures [14].

iv) For all patients with a new diagnosis of epilepsy, the 2017 ILAE position papers on classification of seizures and epilepsy were applied. Seizures were therefore classified as focal onset, generalised onset or unknown onset and further descriptors were applied where possible [11]. Epilepsy was classified as focal, generalised, focal and generalised or unknown depending on available data [12].

\section{Case Ascertainment}

We broadly divided our case ascertainment methods into "hot pursuits", where information was gathered prospectively on a daily basis from active inpatient databases and services, and "cold pursuits" where data were periodically reviewed retrospectively and cases were cross-referenced to the study database to prevent duplication. For an overview of the process of case ascertainment and case analysis, please see Figure 1.

\section{"Hot pursuits"}

i) Emergency departments. Triage recording systems vary in each of the hospitals and therefore it was necessary to screen emergency departments in different ways depending on the resources available. In the main university hospital of the city, electronic triage records allowed us to screen all patients presenting acutely to the emergency department and to identify all possible seizures. The electronic triage system was checked on a daily basis. Search terms including "seizure", "epilepsy", "collapse", "seizure-like events" and "fits" were screened for among the initial post-triage electronic entries. The remaining hospitals operate paper based triage systems and a point of contact in each hospital was established to collect medical record numbers by hand of all patients presenting with a possible seizure.

ii) Radiology. The clinical indication for all CT and MRI brains performed in each of the included hospitals was reviewed systematically to capture all brain imaging requested for an indication of possible seizure. We included direct queries of "first seizure" as well as more indirect queries such as "collapse with jerking".

iv) Inpatient services. Inpatient services with a high likelihood of assessing patients with first seizures were identified. These included neurology, geriatrics, oncology and neurosurgery inpatient services. These services were specifically targeted as it was felt that they might assess and treat patients for a possible seizure without necessarily ordering specific neurophysiology or neuroimaging investigations. These teams were contacted on a fortnightly basis in order to prompt recall of recent cases.

v) Clinical nurse specialists (CNSs). CNSs from services felt to have a high likelihood of encountering patients with a first seizure were contacted on a monthly basis and asked to inform the study team of any new case. CNS in paediatrics, neurology and oncology participated in this study.
"Cold pursuits"

i) EEG databases. There are 3 neurophysiology departments in the capture region, 2 public and one private. All paediatric and adult EEG are performed at one of these 3 sites. The clinical indication for all EEGs performed during the study period was reviewed and all possible first seizures and new diagnosis of epilepsy were included.

ii) RASC. One consultant epileptologist works in the public service in the capture region (author D.J.C.). This consultant runs a RASC once per week with an aim of rapidly reviewing GP and emergency department referrals for "query seizure". All new referrals to this clinic were reviewed on a weekly basis.

iii) Survey of hospital consultants. Consultant physicians in neurology and geriatrics departments in the 7 hospitals outlined above were contacted via postal survey every $8-12$ weeks and asked to alert us if they were aware of any new case.

iv) Hospitals outside of the capture region. We acknowledge the possibility that a small proportion of patients whose residence is toward the outer edge of the capture region may present acutely to a regional hospital outside of the county. There are 3 acute hospitals in the neighboring counties with 4 consultant neurologists working between them. Each neurologist was contacted via a postal survey every 8-12 weeks and asked to alert us if they were aware of any new case which met our inclusion criteria.

v) General practice. All GPs in the defined region were contacted via postal survey every 12 weeks and asked to alert us if s/he was aware of any new case.

vi) Nursing home and residential care survey. Clinical Nurse Manager of all registered nursing homes and residential care services were contacted via postal survey every 6 months and asked to alert us if s/he was aware of any new case. A 6-month interval was chosen for nursing homes as case turnover is much lower than in general practice and we felt that recall would therefore be longer.

vii) Epilepsy Ireland. Epilepsy Ireland is a non-profit, nationwide patient advocacy and advice organisation for people with a diagnosis of epilepsy (www.epilepsyireland.ie). In order to rigorously identify any patient who may be resident in the defined area but who was diagnosed outside of one of the included hospitals, we contacted the local branch of Epilepsy Ireland. They reviewed their new patient database to identify patients who were ordinarily resident in the defined area but were diagnosed elsewhere. An Epilepsy Ireland staff member then consented the patient for inclusion in the study and forwarded their details to the study team.

Of note, we deliberately did not choose pharmacy data on AED prescribing or hospital coding databases for case ascertainment because in Ireland these databases are not linked to other medical data and typically do not discriminate between new cases and established cases of epilepsy.

\section{Identifying under Ascertainment}

As a method of internal control, to determine if we were missing potential cases, we devised a method of assessment of case under ascertainment. We identified that patients with a known diagnosis of primary or secondary brain tumors are at increased risk of seizures [19]. We prospectively collected the medical record numbers of patients discussed at the multidisciplinary neuro-oncology meeting of one tertiary referral university hospital from January to March 2017. Six months later, at the end of September 2017, we searched the medical records of these patients to identify if any had presented with a seizure. For any patient who had a seizure, 


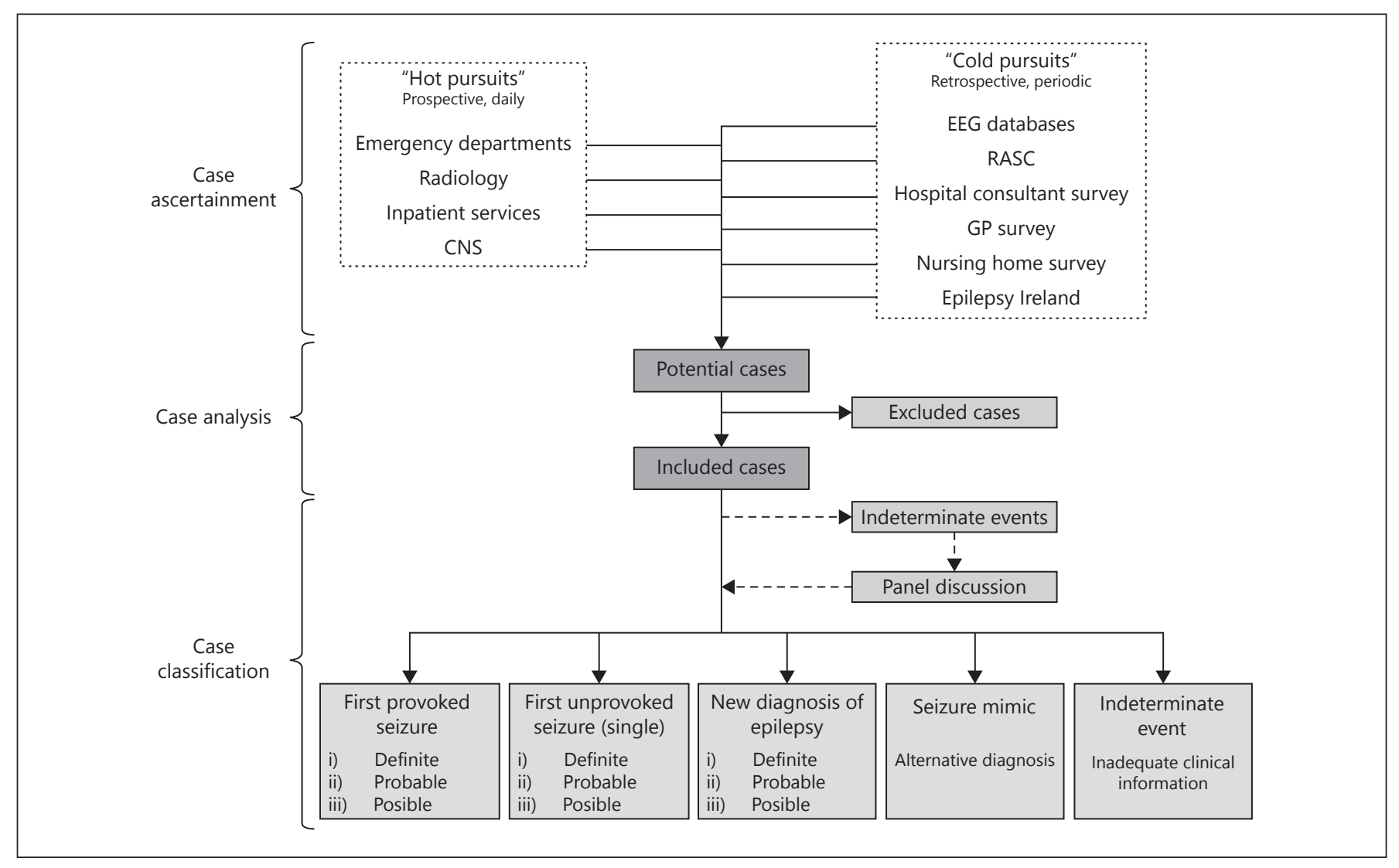

Fig. 1. Overview of case ascertainment, case analysis and case classification protocol. CNS, Clinical nurse specialist; EEG, electroencephalogram; RASC, rapid access seizure clinic; GP, general practitioner.

we then crossed referenced them to our main database to see if they had already been captured through another method of ascertainment.

\section{Case Analysis}

Demographic Data and Clinical Data

The paper medical record of each patient identified through the above methods was obtained from the medical records department. The first author (E.M.M.) abstracted the demographic details, clinical admission details and results of all relevant investigations of each case through medical chart review. For any patient who was reviewed and diagnosed by a consultant neurologist, we adhered to the final diagnosis of the consultant neurologist. For all patients who were not reviewed by a consultant neurologist, the case was analysed by E.M.M. and D.J.C. and a consensus decision was made. Any patient without a clear consensus was further analysed at panel review with a second consultant epileptologist (E.C.). D.J.C. and E.C. are trained neurologists who completed epilepsy fellowships and have $>9$ years clinical experience running epilepsy services.

\section{Case Classification}

Following record review, patients were classified as one of the following 5 categories; first provoked seizure, first single unprovoked seizure, new diagnosis of epilepsy, seizure mimic or indeter- minate event (Fig. 1). Individuals with a first single unprovoked seizure were classified as new diagnosis of epilepsy when it was estimated that there was a $>60 \%$ chance of a recurrent epileptic seizure in the next 10 years, as per the ILAE operational definition of epilepsy [2]. Otherwise, they remained classed as first unprovoked seizure. For definite new cases of epilepsy, the seizure and epilepsy type were subclassified as focal, generalized or unknown according to the ILAE 2017 classification system [11, 12]. Cases were classified as indeterminate, following discussion panel review, if there was insufficient clinical information to accurately classify the case.

The 2011 ILAE Epidemiology Commission report on standards for epidemiologic studies and surveillance of epilepsy [4] provides a classification system for the level of evidence available to support a diagnosis of an epileptic seizure, or new diagnosis of epilepsy, based on the level of epidemiologic evidence available. Based on these standards, the following classification regarding level of evidence of both single epileptic seizures and new diagnosis of epilepsy were applied:

i) Definite. With primary documentation of (a) epileptic seizures, with evidence that these were unprovoked by any acute medical condition or transient brain disorder or (b) documentation of diagnosis by someone with appropriate specialised training in the recognition of epilepsy. Clear evidence of epileptic seizures was most often based on documented collateral history or docu- 


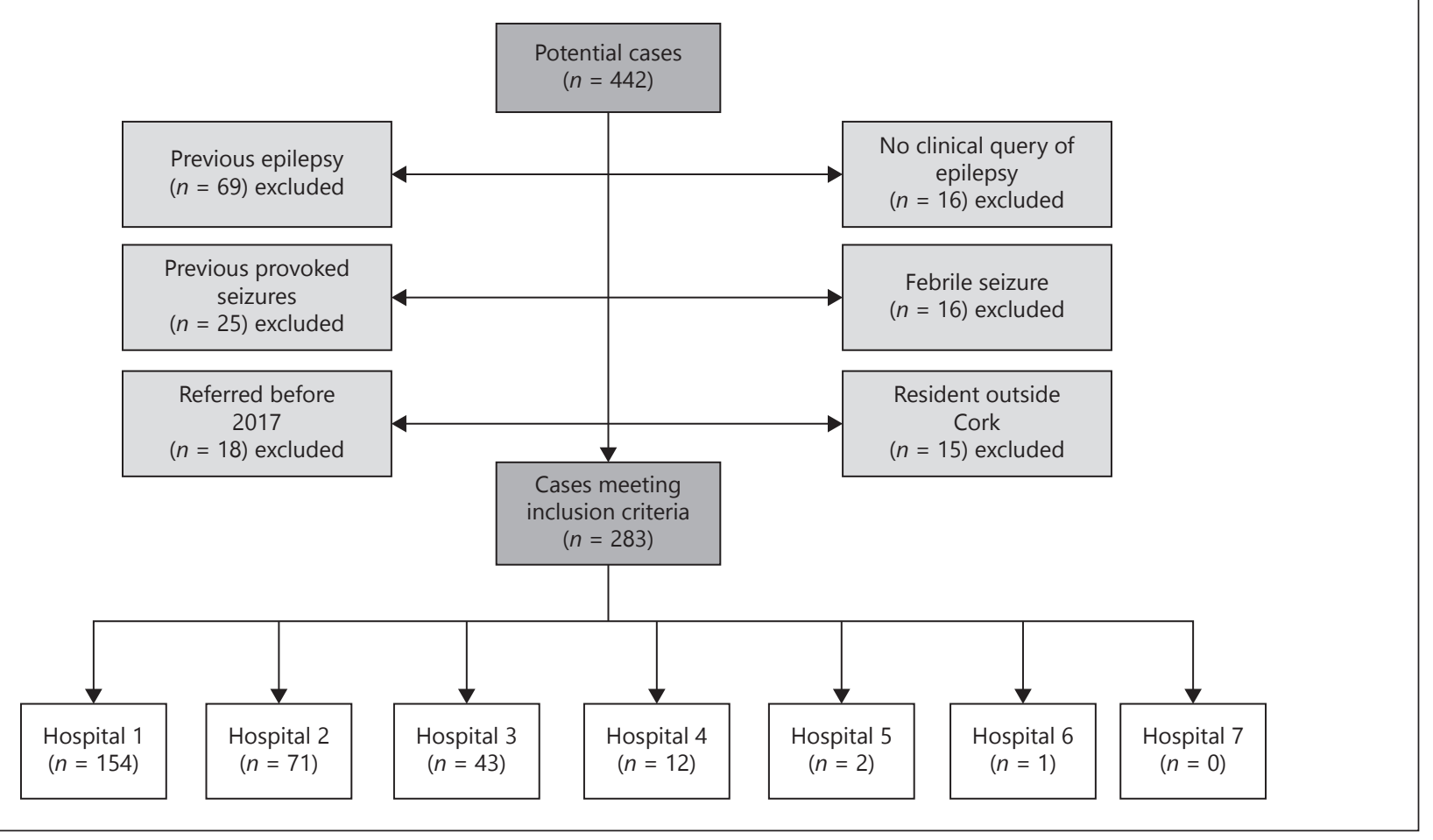

Fig. 2. Flow diagram of identification of potential cases of first seizure and newly diagnosed epilepsy in the capture region over the study period January 1 to March 31, 2017.

mented history by medical staff in the case on inpatient events. In the event of a single documented seizure, evidence of an approximate $60 \%$ risk of recurrence was determined based on the presence or absence of risk factors for recurrence such as epileptiform abnormality on EEG or significant brain imaging abnormality [2022].

ii) Probable. With other sources of information indicating the likelihood that criterion (a) or (b) above is met. For example, cases were defined as probable where there was a history strongly suggestive of an epileptic seizure, but a witnessed history was not recorded.

iii) Suspect. where primary or other sources of information suggest a possibility of epilepsy but neither (a) or (b) above is met. Possible epileptic seizures were defined as an event of which an epileptic seizure was one of a number of plausible differential diagnoses, but of which it was not possible to determine which was the most likely.

Accuracy of Case Information and Classification

In order to ensure the data obtained from the medical records on each case was accurate and consistent, an internal audit of the data extraction was performed (by E.C.). Thirty-eight items were recorded from 3 randomly selected charts. Out of these 114 items $(3 \times 38), 3$ items were discordant between E.C. and E.M.M. data extraction, resulting in a $97.4 \%$ concordance. None of the 3 discordant items altered the clinical diagnosis.

In order to ensure reliability of case classification, ten randomly selected cases were reviewed (by E.C.). In the final classification,
9 of the 10 cases were in concordance. However, the internal audit highlighted the subjectivity of the ILAE practical diagnosis of epilepsy guidelines. In discussion, 6 of the 10 cases did not undoubtedly meet this criterion, and while all team neurologists agreed that these patients would be commenced on an AED due to risk of further seizures, whether this risk reached the $60 \%$ threshold was open to debate.

\section{Results}

The above protocol was applied to our study population from January 1 to March 31, 2018 in order to capture all patients who presented to medical services with a suspected first seizure or new diagnosis of epilepsy during the calendar year 2017. To demonstrate the protocol application, we present the results of the first 3 months of case ascertainment, January 1, 2017 to March 31, 2017.

A flow diagram of the potential cases of first seizure or newly diagnosed epilepsy from January 1 to March 31, 2017 is shown in Figure 2. Four hundred and forty 2 cases were initially identified. Following review of patient charts, 159 (40\%) were excluded as they did not meet the inclusion criteria. The most common reason for exclusion was previously diagnosed epilepsy $(n=69,16 \%)$ or 
Fig. 3. Pie chart demonstrating overlapping sources of hospital based case ascertainment during study period January 1 to March 31, $2017(n=283)$. The chart shows the proportion of included cases identified uniquely by each of the 6 hospital based methods as well as the proportion of cases identified by 2, 3 or $>3$ individual sources. CNS, clinical nurse specialist; ED, emergency department; EEG, electroencephalogram; RASC, rapid access seizure clinic.

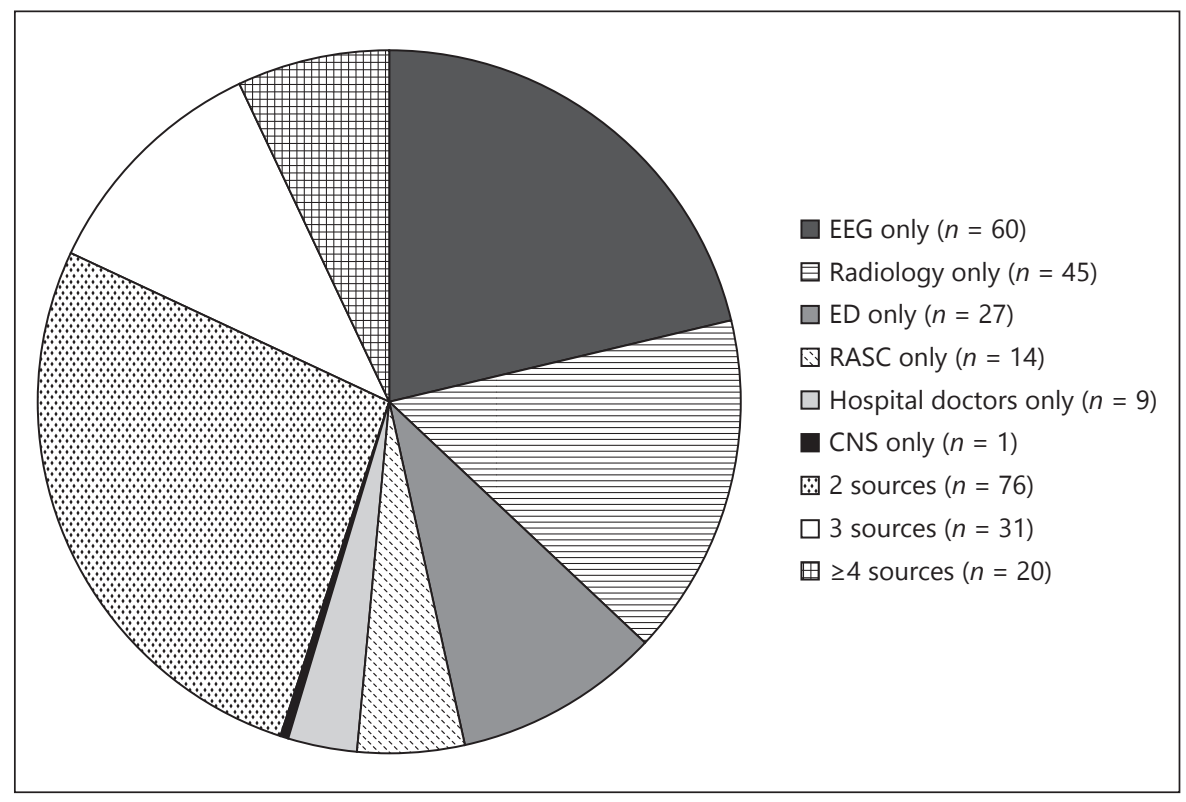

Table 2. Cases identified at each source expressed to the nearest percentage

All included potential first seizure or new diagnosis epilepsy patients $(n=283)$

\begin{tabular}{lcc}
\hline source & $\begin{array}{l}\text { number of } \\
\text { cases }(\%)\end{array}$ & $\begin{array}{l}\text { unique to } \\
\text { source, } n(\%)\end{array}$ \\
\hline Radiology & $153(54)$ & $45(16)$ \\
EEG & $151(53)$ & $60(21)$ \\
Emergency department & $70(25)$ & $27(9)$ \\
Hospital doctors & $53(19)$ & $9(3)$ \\
RASC & $45(16)$ & $14(5)$ \\
Community survey & $16(6)$ & $0(0)$ \\
CNS & $11(4)$ & $1(<1)$ \\
Epilepsy Ireland & $0(0)$ & $0(0)$ \\
\hline
\end{tabular}

EEG, electroencephalogram; RASC, rapid access seizure clinic; CNS, clinical nurse specialist.

recurrent provoked seizures $(n=25,6 \%)$. Two hundred and eighty- 3 patients were included and were distributed between 6 of the 7 hospitals in Cork city and county.

A pie chart illustrating the overlapping hospital-based sources of case ascertainment is shown in Figure 3. Of the 283 included cases, 55 percent $(n=156)$ were identified by a single source of case ascertainment, $27 \%(n=76)$ were identified by 2 sources, $11 \%(n=31)$ by 3 sources and $7 \%(n=20)$ were identified by $>3$ sources of ascertainment. No single case was identified by all of the meth-

Methodology of a Prospective Incidence Study on Epilepsy, Seizures and Mimics ods of case ascertainment. Regarding the contribution of individual sources of case ascertainment, almost $54 \%$ of cases were identified by reviewing radiology databases, see Table 2. The highest number of cases that was "unique to source", meaning not identified elsewhere, came from review of EEG databases.

Figure 4 illustrates the first round of postal surveys of GPs in the defined area. The response rate was $58 \%$. Five GPs wrote back to indicate that they were retired and were therefore excluded from future surveys. Twentyfour potential cases were identified through this postal survey; however 8 were subsequently excluded as they did not meet the inclusion criteria. Sixteen cases were included in the study, none of which was unique to the GP survey in terms of source of case ascertainment. The community based patient advocacy group Epilepsy Ireland reviewed their records and did not yield any new cases diagnosed outside of a Cork hospital in the first 3 months of cases ascertainment.

Figure 5 illustrates the break-down of identified patients into each of the 5 subclassifications- first provoked seizure, first unprovoked seizure, new diagnosis of epilepsy, seizure mimics and indeterminate. Among the 95 cases of new diagnosis of epilepsy, 75 (79\%) were patients who had a first seizure in 2017, and had an estimated $>60 \%$ chance of further seizures, therefore met the criteria for a practical definition of epilepsy [2]. Seventy-one of the new diagnosis of epilepsy were considered definite, and of these $71 \%(\mathrm{n}=50)$ were focal, $18 \%(n=13)$ were generalized and in $11 \%(n=8)$ cases it was unknown 
Fig. 4. Flow diagram illustrating case ascertainment by postal survey of all GPs in the capture region during study period January 1 to March 31,2017. A total of 16 cases were included from this method of ascertainment, all of whom had been previously identified through hospital based ascertainment. GP, general practitioner.
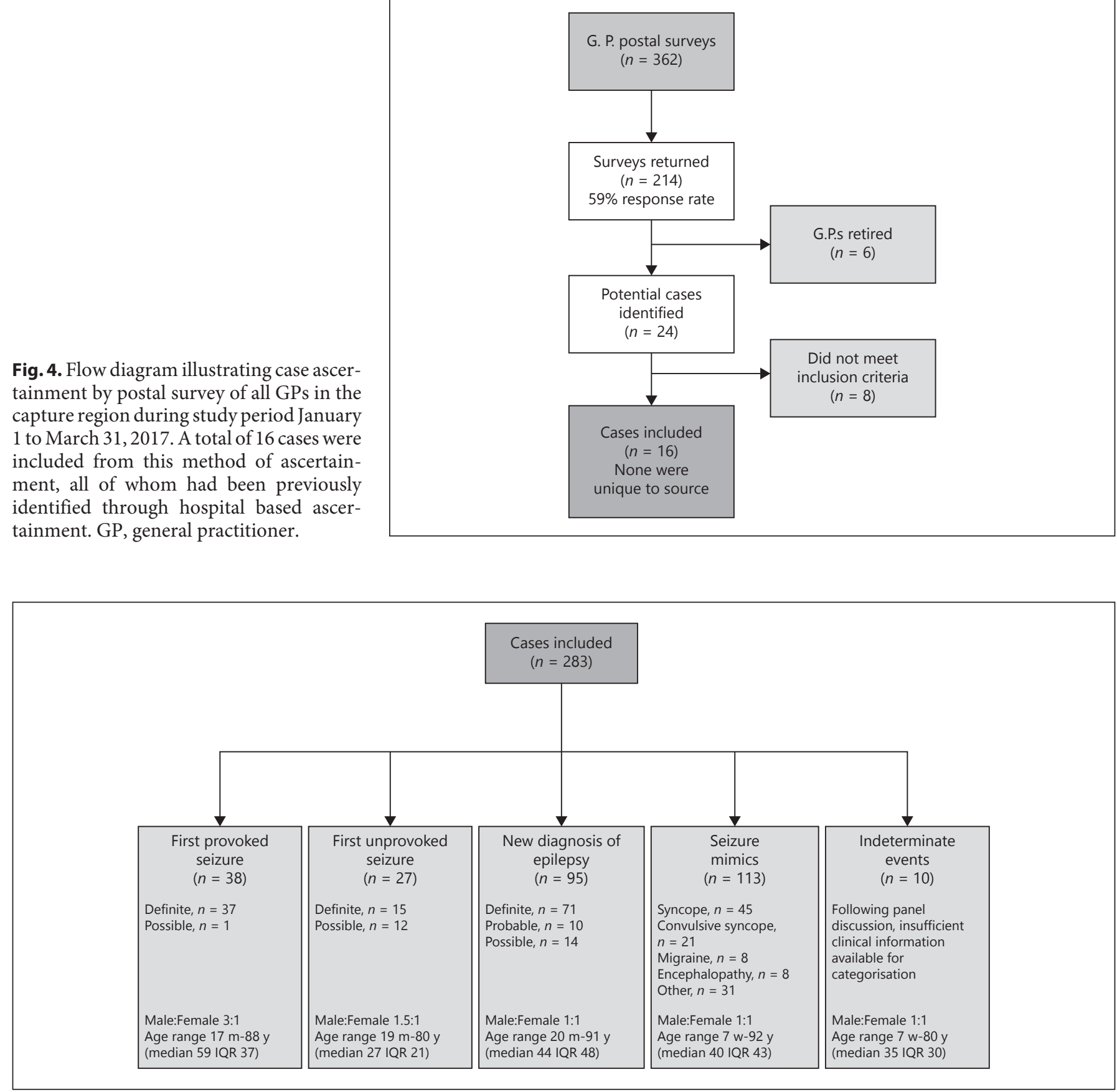

Fig. 5. Number of cases in each subgroup following panel review of case information. Of the 95 cases of new diagnosis of epilepsy, 75 also presented with their first seizure and were determined to be at $>60 \%$ risk of recurrent seizures. IQR, interquartile range; $m$, months; w, weeks; $y$, years.

whether epilepsy was focal or generalized according to the ILAE 2017 subclassification of seizure and epilepsy type. Seizure mimics represented the largest group $(n=$ $113,40 \%$ of all reviewed case records) and the most commonly encountered seizure mimic was syncope $(40 \%, n=$
45). There were $10(3 \%)$ indeterminate cases in the first 3 months of data collection. Extrapolating the first 3 months of data, the crude incidence for new diagnosis of epilepsy in our population was 59 per 100,000 people per year, however it is necessary to highlight that this is an esti- 
Table 3. Comparison of case ascertainment methods in previous studies investigating the incidence of epilepsy and/or first seizures in all ages within a defined population

\begin{tabular}{|c|c|c|c|c|c|c|c|c|c|c|}
\hline Study & $\begin{array}{l}\text { Current } \\
\text { study }\end{array}$ & $\begin{array}{l}\text { Olafsson } \\
\text { et al. [23], } \\
2005\end{array}$ & $\begin{array}{l}\text { MacDonald } \\
\text { et al. [24], } \\
2000\end{array}$ & $\begin{array}{l}\text { Jallon } \\
\text { et al. [25], } \\
1999\end{array}$ & $\begin{array}{l}\text { Jallon } \\
\text { et al. [26], } \\
1997\end{array}$ & $\begin{array}{l}\text { Loiseau } \\
\text { et al. [9], } \\
1990\end{array}$ & $\begin{array}{l}\text { Joensen } \\
{[27], 1986}\end{array}$ & $\begin{array}{l}\text { Granieri } \\
\text { et al. [28], } \\
1983\end{array}$ & $\begin{array}{l}\text { Hauser } \\
\text { and Kurland } \\
{[29], 1975}\end{array}$ & $\begin{array}{l}\text { de Graaf } \\
{[30], 1974}\end{array}$ \\
\hline Geographic area (population) & $\begin{array}{l}\text { Cork, } \\
\text { Ireland, } \\
(542,868)\end{array}$ & $\begin{array}{l}\text { Iceland, } \\
(882,151)\end{array}$ & $\begin{array}{l}\text { London, } \\
\text { United } \\
\text { Kingdom, } \\
(100,230)\end{array}$ & $\begin{array}{l}\text { Martinique } \\
\text { Island } \\
(383,596)\end{array}$ & $\begin{array}{l}\text { Genevea, } \\
\text { Switzerland } \\
(384,657)\end{array}$ & $\begin{array}{l}\text { Southwest } \\
\text { France } \\
(1,128,164)\end{array}$ & $\begin{array}{l}\text { Faroe } \\
\text { Island } \\
(41,144)\end{array}$ & $\begin{array}{l}\text { Copparo, } \\
\text { Italy } \\
(45,153)\end{array}$ & $\begin{array}{l}\text { Rochester, } \\
\text { United States }\end{array}$ & $\begin{array}{l}\text { Northern } \\
\text { Norway } \\
(215,000)\end{array}$ \\
\hline
\end{tabular}

Case ascertainment Radiology database RASC

CNS EEG database

Emergency department Neurologists/pediatricians Other hospital specialists

GP survey

Residential care survey

Patient advocacy group

Hospital medical record data

School teachers and social workers

Incidence of first unprovoked

seizures (per 100,000)

Incidence of first provoked

seizures (per 100,000)

Incidence of epilepsy (per 100,000)

$\mathrm{X}$
$\mathrm{X}$
$\mathrm{X}$
$\mathrm{X}$
$\mathrm{X}$
$\mathrm{X}$
$\mathrm{X}$
$\mathrm{X}$
$\mathrm{X}$
$\mathrm{X}$

$\mathrm{X}$
$\mathrm{X}$
$\mathrm{X}$
$\mathrm{X}$
$\mathrm{X}$

$\mathrm{X}$
$\mathrm{X}$

\begin{tabular}{|c|c|c|c|}
\hline 56.8 & 11 & 64.1 & 45.6 \\
\hline & & 16.4 & 25.2 \\
\hline
\end{tabular}

$\mathrm{x}$

$\mathrm{X}$

$\mathrm{X}$

$\mathrm{X}$
$\mathrm{X}$

$\mathrm{X}$

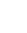

$x^{2}$

$\mathrm{X}$

$\mathrm{X}$

EEG, electroencephalogram; RASC, rapid access seizure clinic; CNS, clinical nurse specialist; GP, general practitioner. mated rate which is likely to increase as it does not allow for "late entries" due to lag in case ascertainment methods. The incidence is higher than previous studies outlined in Table 3 and therefore reinforces the importance of multiple methods of case ascertainment.

To estimate potential case under-ascertainment, 63 patients were identified by screening 3 months of multidisciplinary neuro-oncology meetings at a tertiary referral center. Of these, 5 had a previous diagnosis of epilepsy and 36 were not resident in the defined area therefore were not eligible for inclusion in the study. The medical charts of the remaining 22 patients were reviewed and none had a documented seizure during follow-up.

\section{Discussion}

We present an epidemiological protocol that adheres to recently published international guidelines for investigation of the incidence of epilepsy within a population [4, 10]. Furthermore, we have incorporated recently updated ILAE guidelines for the clinical classification of seizures and epilepsy $[11,12]$ which have not yet been used in epidemiological studies but which will be of increasing importance in international epilepsy research. Our case ascertainment protocol is rigorous and, when data analysis is complete, will be the first to report the incidence of epilepsy, first unprovoked seizures and first provoked seizures in Ireland. Finally, to our knowledge, this will be the first study to additionally report the incidence and characteristics of "seizure mimics" within the same population that seizures and epilepsy are under study. Inclusion of the related diagnosis of first seizures, epilepsy and seizure mimics in our study protocol gives a sense of the scale of this cohort and the significant impact on healthcare services investigating and treating these patients. Further details on age-adjusted incidence, seizure and epilepsy etiology, and classification will be reported separately when annual incidence data is complete.

Many previous epidemiologic studies have focused on a specific cohort within a population, for example children or the elderly. Relatively few have included all age-groups and, within those studies, all available case ascertainment methods range from use of a single source to multiple overlapping sources, see Table 3. With regard to case ascertainment in our study, we aimed for full capture of new events by combining as many methods of ascertainment as was practicable in 
our population. As shown in Table 3, the majority of other studies do not use such an extensive combination of methods. For example, Jallon et al. [27], used EEG database as the sole method of case ascertainment. In our study, EEG databases identified only $53 \%$ of cases. Furthermore, our interim 3-month analysis demonstrates the importance of including all possible methods of ascertainment as no single case was picked up in every method. In addition, we specifically targeted inpatient teams and CNSs with a high likelihood of encountering a patient with a first seizure, for example, the neurosurgical and oncology teams, and contacted them on a regular basis to maximize case ascertainment. These methods identified 10 "unique to source" cases, not identified by other methods in the first 3 months of case ascertainment. To our knowledge, this is the first study to apply such a method.

The seminal work by Hauser and Kurland [29], in Rochester Minnesota demonstrates the usefulness of accurate medical record databases to perform epidemiologic studies. However, such medical record linkage systems are not available in many countries, including Ireland, and therefore in order to obtain accurate epidemiologic data overlapping methods of case ascertainment are required. Data obtained from cohort specific databases were used by Annegers et al. [30], to describe the incidence of epilepsy in a multiethnic urban population (35.5 per 100,000). However, this database contained information only on those enrolled in a health maintenance organization served by a particular clinic and was therefore findings may not be generalizable to the whole population as the clinic served only people in employment and their dependents. Correspondingly, this study demonstrated low incidence among those over 65 years of age, in contrast to most other studies. Therefore, in populations where accurate, whole population, medical record databases are not in existence, multiple over lapping methods of ascertainment, in combination with medical chart review, as demonstrated by our study, provide detailed information for case identification, classification and determination of etiology.

An early study in Italy [28] was unique in its use of social workers and teachers in the community to maximize case ascertainment, see Table 3. However, due to ethical and practical considerations this would no longer be a viable source of case ascertainment. Finally, studies carried out in lower income countries, with less well developed health resources, have used door-to-door community surveys to ascertain cases, for example [32,33]. However, this method is vulnerable to under-ascertainment if stigma is associated with epilepsy and seizures in the population. Furthermore, accuracy of diagnosis and classification of cases is difficult when this method is used in isolation. Therefore, when adequate health resources permit, such as in our study, the use of hospital-based databases to identify and classify patients based on clinical information is preferable.

Other potential sources of case ascertainment were also considered. Previously published studies investigating adult populations have used AED prescription databases as a source of case ascertainment [34, 35]. However, it was not possible to use AED prescribing databases in our case ascertainment for a number of reasons. Firstly, drug prescribing data are not linked to other patient records in our population, therefore it would not have been possible to cross reference such information to our database. Secondly, patients presenting with a first seizure or seizure mimic may not routinely be prescribed an AED and therefore would not be captured by such a method. Thirdly, AEDs are often used for indications other than epilepsy, such as neuropathic pain, and therefore may overestimate the diagnosis of epilepsy. Finally, use of a drug prescription database over the course of a year, without linkage to other patient records, would not give an accurate account of patients who were newly prescribed the medication. Therefore, in our population, such a method would be more appropriately applied to estimating prevalence of epilepsy rather than incidence, as was demonstrated by Linehan et al. [13].

Very few studies address under-ascertainment. MacDonald et al. [24] cross-referenced general practice databases with local hospital patient administration system to try to ensure complete ascertainment. However, this method relies on accurate and complete case coding. For a number of patients, a seizure may not be the primary reason for attending hospital, for example, patients incorrectly initially diagnosed as a stroke, or patients who have their first seizure while medically unwell for another reason. To our knowledge, our study is the first to design a unique method of following "at risk" patients as a method of assessment of under-ascertainment. However, for the first 3 months of data collection, none of these at risk patients proceeded to have a seizure. We propose to follow this cohort for a longer time-frame in order to determine under-ascertainment and validate this unique method. On the other hand, it is reassuring that no community obtained case (from general practice or nursing homes) had not been identified already using the hospital-based meth- 
ods, and therefore this serves as somewhat of a surrogate of complete ascertainment.

Our study highlighted the subjectivity involved in determining whether, following a single seizure, a person is at $>60 \%$ risk of further seizures, as found by our panel discussion. The Multicenter Trial for Early Epilepsy and Single Seizures [36] provides some guidance on assessing this risk. However, the Multicenter Trial for Early Epilepsy and Single Seizures study did not include neuroimaging as part of its risk stratification tool and there remains a lack of up-to-date, real world clinical data in certain populations, for example, older adults with established small vessel ischaemic disease with juxtacortical signal changes on brain imaging. In clinical practice, many patients commence an AED because they are considered at some risk of recurrent events, even though the exact risk is unquantifiable. The 2014 ILAE practical definition of epilepsy does not require the clinician to quantify the risk precisely. Therefore, we have included patients within the cohort of new diagnosis of epilepsy who were determined to be at an approximate $60 \%$ risk of recurrent seizures based on the evidence available to date. Further study of individuals at the margin of this risk threshold is required.

We are aware of potential gaps in case ascertainment, for example people who did not seek medical help after a seizure, people who do not recognize that they have had a seizure(s) and vulnerable patients unable to access medical care including homeless people, non-verbal individuals and people living alone. In addition, epilepsy and mimics largely are based on clinical history. In a small number of cases the treating physician may not recognize seizure as a potential diagnosis. However, through our study design, we have maximized case ascertainment in as much as possible.

\section{Acknowledgement}

The authors would like to thank all hospital-based medical, nursing, neurophysiology, radiology and medical records administrative staff as well as community-based GPs and nursing home staff who contributed and responded to the study. We would also like to thank Dr. Paul Corcoran (School of Public Health, University College Cork) and Dr. Carol Sinnott for advice during study design.

\section{Statement of Ethics}

Ethical approval was obtained from the Clinical Research Ethics Committee of the Cork Teaching Hospitals. This was an observational study and following collection, all data were anonymized and stored on a password-protected computer in the Department of Neurology, Cork University Hospital.

\section{Disclosure Statement}

The authors have no conflicts of interest to declare.

\section{Funding sources}

No specific funding was received by any author with regard to this study.

\section{Author Contributions}

E.M.M. was involved in study design, data collection, data analysis and manuscript preparation. E.C. was involved in study design, data analysis and manuscript preparation. É.J.O.R. was involved in study design, data analysis and manuscript preparation. D.J.C. was involved in study design, data collection, analysis and manuscript preparation.

\section{References}

1 Fisher RS, van Emde Boas W, Blume W, Elger C, Genton P, Lee P, et al. Epileptic seizures and epilepsy: definitions proposed by the International League Against Epilepsy (ILAE) and the International Bureau for Epilepsy (IBE). Epilepsia. 2005 Apr;46(4):470-2.

2 Fisher RS, Acevedo C, Arzimanoglou A, Bogacz A, Cross JH, Elger CE, et al. ILAE official report: a practical clinical definition of epilepsy. Epilepsia. 2014 Apr;55(4):475-82.

3 Allers K, Essue BM, Hackett ML, Muhunthan J, Anderson CS, Pickles K, et al. The economic impact of epilepsy: a systematic review. BMC Neurol. 2015 Nov;15(1):245.
4 Thurman DJ, Beghi E, Begley CE, Berg AT, Buchhalter JR, Ding D, et al.; ILAE Commission on Epidemiology. Standards for epidemiologic studies and surveillance of epilepsy. Epilepsia. 2011 Sep;52 Suppl 7:226.

5 Banerjee PN, Filippi D, Allen Hauser W. The descriptive epidemiology of epilepsy-a review. Epilepsy Res. 2009 Jul;85(1):31-45.

6 Fiest KM, Sauro KM, Wiebe S, Patten SB, Kwon CS, Dykeman J, et al. Prevalence and incidence of epilepsy: A systematic review and meta-analysis of international studies. Neurology. 2017 Jan;88(3):296-303.
7 Ngugi AK, Bottomley C, Scott JA, Mung'alaOdera V, Bauni E, Sander JW, et al. Incidence of convulsive epilepsy in a rural area in Kenya. Epilepsia. 2013 Aug;54(8): 1352-9.

8 Cesnik E, Pedelini F, Faggioli R, Monetti VC, Granieri E, Casetta I. Incidence of epilepsy in Ferrara, Italy. Neurol Sci. 2013 Dec;34(12): 2167-72.

9 Loiseau J, Loiseau P, Guyot M, Duche B, Dartigues JF, Aublet B. Survey of seizure disorders in the French southwest. I. Incidence of epileptic syndromes. Epilepsia. 1990 Jul-Aug; 31(4):391-6.
Methodology of a Prospective Incidence Study on Epilepsy, Seizures and Mimics 
10 Bennett DA, Brayne C, Feigin VL, BarkerCollo S, Brainin M, Davis D, et al. Development of the Standards of Reporting of Neurological Disorders (STROND) checklist: A guideline for the reporting of incidence and prevalence studies in neuroepidemiology. Neurology. 2015 Sep;85(9):821-8.

11 Fisher RS, Cross JH, French JA, Higurashi N, Hirsch E, Jansen FE, et al. Operational classification of seizure types by the International League Against Epilepsy: Position Paper of the ILAE Commission for Classification and Terminology. Epilepsia. 2017 Apr;58(4):52230.

12 Scheffer IE, Berkovic S, Capovilla G, Connolly MB, French J, Guilhoto L, et al. ILAE classification of the epilepsies: Position paper of the ILAE Commission for Classification and Terminology. Epilepsia. 2017 Apr;58(4):51221.

13 Linehan C, Kerr MP, Walsh PN, Brady G, Kelleher C, Delanty N, et al. Examining the prevalence of epilepsy and delivery of epilepsy care in Ireland. Epilepsia. 2010 May;51(5): $845-52$.

14 Beghi E, Carpio A, Forsgren L, Hesdorffer DC, Malmgren K, Sander JW, et al. Recommendation for a definition of acute symptomatic seizure. Epilepsia. 2010 Apr;51(4):671-5.

15 Labovitz DL, Hauser WA, Sacco RL. Prevalence and predictors of early seizure and status epilepticus after first stroke. Neurology. 2001 Jul;57(2):200-6.

16 Camilo O, Goldstein LB. Seizures and epilepsy after ischemic stroke. Stroke. 2004 Jul; 35(7):1769-75.

17 Hesdorffer DC, Benn EK, Cascino GD, Hauser WA. Is a first acute symptomatic seizure epilepsy? Mortality and risk for recurrent seizure. Epilepsia. 2009 May;50(5):1102-8.

18 Jennett B, Teather D, Bennie S. Epilepsy after head injury. Residual risk after varying fit-free intervals since injury. Lancet. 1973 Sep; 2(7830):652-3.

19 Vecht CJ, Kerkhof M, Duran-Pena A. Seizure prognosis in brain tumors: new insights and evidence-based management. Oncologist. 2014 Jul;19(7):751-9.

20 Stroink H, Brouwer OF, Arts WF, Geerts AT, Peters AC, van Donselaar CA. The first unprovoked, untreated seizure in childhood: a hospital based study of the accuracy of the diagnosis, rate of recurrence, and long term outcome after recurrence. Dutch study of epilepsy in childhood. J Neurol Neurosurg Psychiatry. 1998 May;64(5):595-600.

21 Bergey GK. Management of a First Seizure. Continuum (Minneap Minn). 2016 Feb;22 1 Epilepsy:38-50.

22 Stösser S, Böckler S, Ludolph AC, Kassubek J, Neugebauer H. Juxtacortical lesions are associated with seizures in cerebral small vessel disease. J Neurol. 2019 May;266(5):1230-5.

23 Olafsson E, Ludvigsson P, Gudmundsson G, Hesdorffer D, Kjartansson O, Hauser WA. Incidence of unprovoked seizures and epilepsy in Iceland and assessment of the epilepsy syndrome classification: a prospective study. Lancet Neurol. 2005 Oct;4(10):62734.

24 MacDonald BK, Cockerell OC, Sander JW, Shorvon SD. The incidence and lifetime prevalence of neurological disorders in a prospective community-based study in the UK. Brain. 2000 Apr;123(Pt 4):665-76.

25 Jallon P, Smadja D, Cabre P, Le Mab G, Bazin M. EPIMART: prospective incidence study of epileptic seizures in newly referred patients in a French Carribean island (Martinique). Epilepsia. 1999 Aug;40(8):1103-9.

26 Jallon P, Goumaz M, Haenggeli C, Morabia A. Incidence of first epileptic seizures in the canton of Geneva, Switzerland. Epilepsia. 1997 May;38(5):547-52.
27 Joensen P. Prevalence, incidence, and classification of epilepsy in the Faroes. Acta Neurol Scand. 1986 Aug;74(2):150-5.

28 Granieri E, Rosati G, Tola R, Pavoni M, Paolino E, Pinna L, et al. A descriptive study of epilepsy in the district of Copparo, Italy, 19641978. Epilepsia. 1983 Aug;24(4):502-14.

29 Hauser WA, Kurland LT. The epidemiology of epilepsy in Rochester, Minnesota, 1935 through 1967. Epilepsia. 1975 Mar;16(1):1-66.

30 de Graaf AS. Epidemiological aspects of epilepsy in northern Norway. Epilepsia. 1974 Sep;15(3):291-9.

31 Annegers JF, Dubinsky S, Coan SP, Newmark ME, Roht L. The incidence of epilepsy and unprovoked seizures in multiethnic, urban health maintenance organizations. Epilepsia. 1999 Apr;40(4):502-6.

32 Tekle-Haimanot R, Forsgren L, Ekstedt J. Incidence of epilepsy in rural central Ethiopia. Epilepsia. 1997 May;38(5):541-6.

33 Medina MT, Durón RM, Martínez L, Osorio JR, Estrada AL, Zúniga C, et al. Prevalence, incidence, and etiology of epilepsies in rural Honduras: the Salamá Study. Epilepsia. 2005 Jan;46(1):124-31.

34 Kaiboriboon K, Bakaki PM, Lhatoo SD, Koroukian $S$. Incidence and prevalence of treated epilepsy among poor health and low-income Americans. Neurology. 2013 May; 80(21):1942-9.

35 Choi H, Pack A, Elkind MS, Longstreth WT Jr, Ton TG, Onchiri F. Predictors of incident epilepsy in older adults: The Cardiovascular Health Study. Neurology. 2017 Feb;88(9): 870-7.

36 Kim LG, Johnson TL, Marson AG, Chadwick DW; MRC MESS Study group. Prediction of risk of seizure recurrence after a single seizure and early epilepsy: further results from the MESS trial. Lancet Neurol. 2006 Apr;5(4): $317-22$. 